\title{
Unusual Presentations of Eccrine Porocarcinomas
}

\author{
Lester Juay $^{\mathrm{a}}$ Ellie Choi ${ }^{\mathrm{a}} \quad$ Jingxiang Huang ${ }^{\mathrm{b}}$ Huma Jaffar $^{\mathrm{a}}$ Sue-Ann Ju Ee Ho ${ }^{c}$ \\ aDepartment of Dermatology, National University Hospital, Singapore, Singapore; ${ }^{b}$ Department of Pathology, \\ Sengkang General Hospital, Singapore, Singapore; 'Dermatology \& Surgery Clinic, Singapore, Singapore
}

\section{Established Facts}

- Eccrine porocarcinomas are rare tumours, albeit the most common malignant adnexal tumours of the skin.

- Eccrine porocarcinomas can present with very heterogeneous clinical and non-distinctive dermoscopic features, rendering diagnosis limited to histopathological examination alone.

\section{Novel Insights}

- Our first case interestingly demonstrates an occurrence of porocarcinoma after the diagnosis of SCC, suggesting the possibility of unknown risk factors for both.

- While eccrine porocarcinomas can demonstrate good response to radiotherapy alone, further research can be done on combination therapy with hormone receptor antagonists.

- Our second case demonstrates malignant change of a poroma - this may be a more common phenomenon than we would expect based on the current literature.

\section{Keywords}

Adnexal tumour · Porocarcinoma · Poroma

\begin{abstract}
Eccrine porocarcinomas (EPCs) are rare tumours, albeit the most common malignant adnexal tumours of the skin. They can present with very heterogeneous clinical and dermoscopic features, rendering diagnosis limited to histopathological examination alone. We share 2 cases of EPCs, one of which arose in a patient with a prior diagnosis of cutaneous squamous cell carcinoma (SCC) and another whose EPC was likely a malignant transformation of an existing poroma. An occurrence of porocarcinoma after the diagnosis of SCC may suggest the possibility of unknown risk factors for both. Positivity to androgen, oes-
\end{abstract}

karger@karger.com www.karger.com/sad

Karger $\stackrel{\text { ' }}{5}$

GOPEN ACCESS
C 2021 The Author(s).

Published by S. Karger AG, Basel

This article is licensed under the Creative Commons Attribution 4.0 International License (CC BY) (http://www.karger.com/Services/ OpenAccessLicense). Usage, derivative works and distribution are permitted provided that proper credit is given to the author and the original publisher. trogen, and epidermal growth factor receptors was seen in a proportion of porocarcinomas, and this may prompt further research on combination therapy between conventional treatment modalities with hormone receptor antagonists. Malignant change of a poroma may be a more common phenomenon than we would expect based on the current literature.

$$
\begin{aligned}
& \text { (C) } 2021 \text { The Author(s). } \\
& \text { Published by S. Karger AG, Basel }
\end{aligned}
$$

\section{Introduction/Literature Review}

Eccrine porocarcinomas (EPCs) are rare tumours, accounting for $0.005-0.01 \%$ of cutaneous tumours [1]. Like other cutaneous adnexal carcinomas, they can present with very heterogeneous clinical and non-distinctive der- 

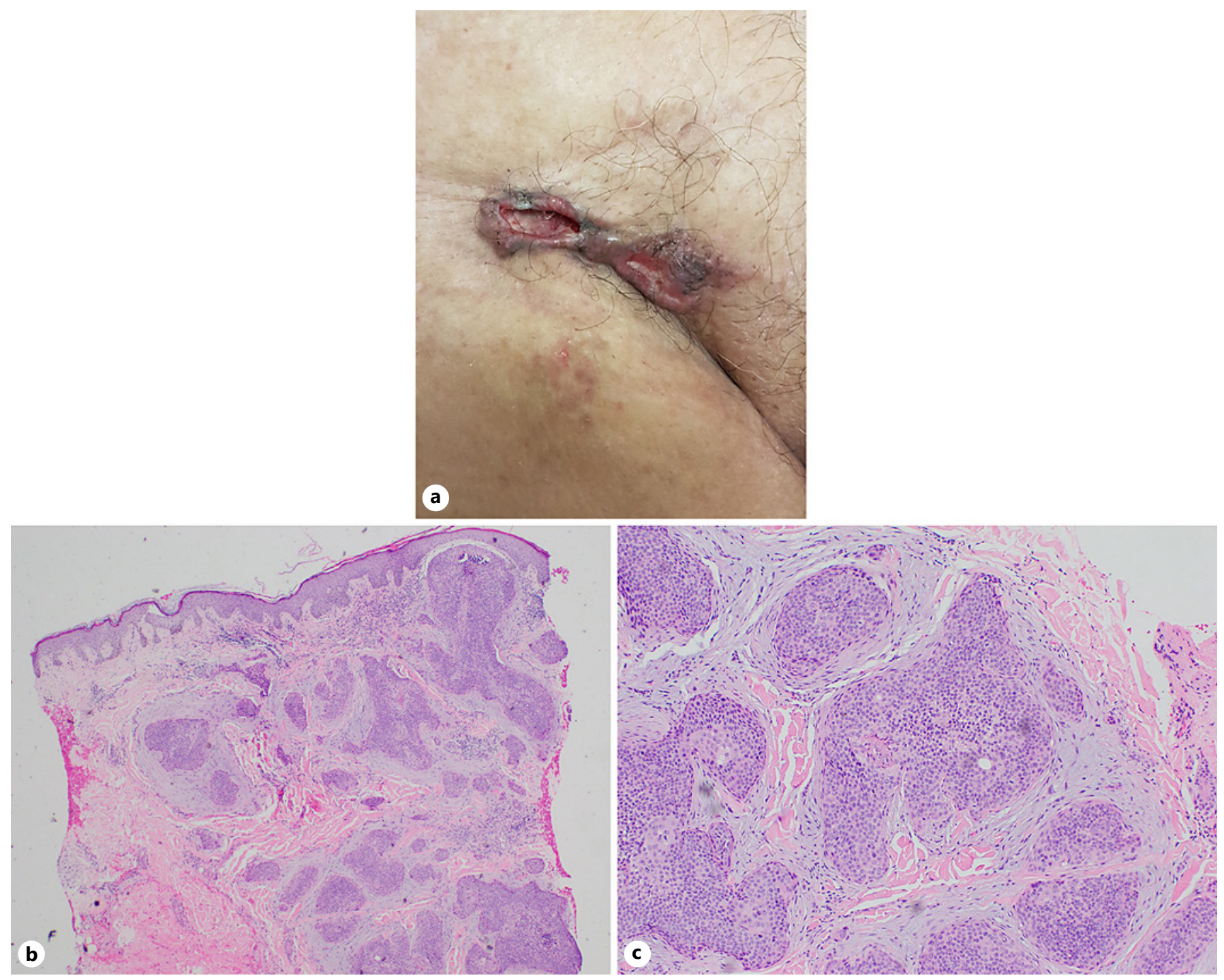

Fig. 1. a Ulcerated plaque over the right groin. b Photomicrograph showing irregular nests of tumour cells in a desmoplastic stroma (H\&E, original magnification, $\times 40)$. c Higher power view showing poroid cells with round to ovoid nuclei and focal duct formation (H\&E, original magnification, $\times 100)$.

moscopic features, rendering diagnosis limited to histopathological examination alone [2]. We discuss the varied presentation of porocarcinomas through the illustration of 2 cases: the first, an elderly patient with de novo porocarcinoma, and the second, a patient with porocarcinoma from malignant transformation.

\section{Case Report/Case Presentation}

The first case is of a 90-year-old Chinese gentleman, who was referred for a progressively enlarging ulcerative plaque over the right groin for 6 months. He had a significant history of a previous squamous cell carcinoma (SCC) of the right shin $>10$ years ago, treated with excision and skin grafting.

Examination revealed a firm ulcerated plaque over the right groin with irregular margins and surface (Fig. 1a). The initial impression was a primary cutaneous carcinoma (such as SCC or amelanotic melanoma), with the differential of ulcerated inguinal lymph nodes. Punch biopsy revealed irregular nests of tumour cells with round to ovoid nuclei and focal duct formation in a desmoplastic stroma (Fig. 1b). Focal areas of calcification, squamous differentiation, and necrosis within tumour nests were seen. Mitotic activity was frequent (Fig. 1c). Immunohistochemical staining was negative for CK19. CEA staining showed areas of ductal formation. This was consistent with a diagnosis of porocarcinoma. 

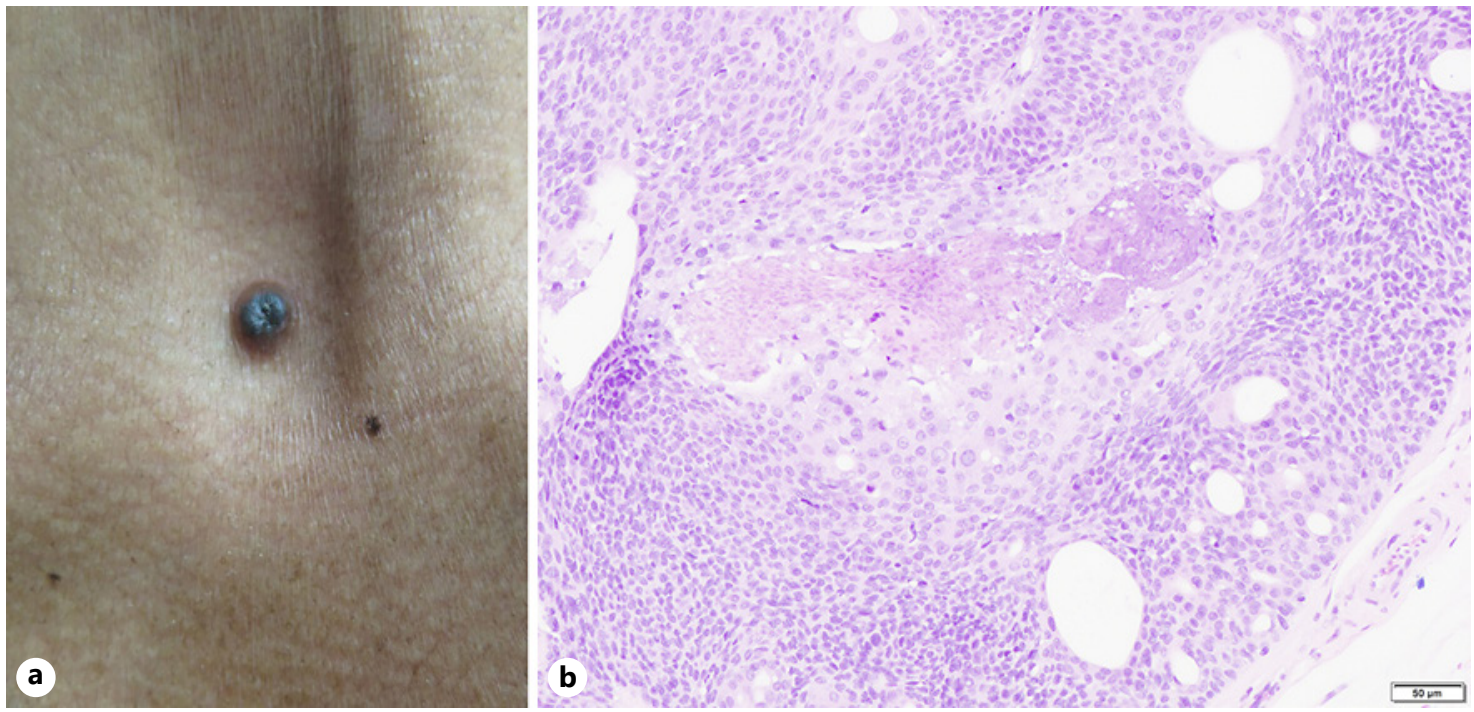

Fig. 2. a Pigmented nodule on the suprasternal notch. b Photomicrograph of nests of cells with ovoid nuclei showing nuclear crowding and moderate nuclear pleomorphism.

A positron emission tomography, done for concerns of nodal and distant metastases, showed a hypermetabolic soft tissue density in the right inguinal region with superficial skin defect, compatible with the ulcer, and hypermetabolic destructive bony lesions in the right ilium, acetabulum, and pubis. Mildly FDGavid fat stranding was seen in the right thigh, suggestive of lymphatic obstruction. A diagnosis of stage IV porocarcinoma was made.

Additional staining for hormonal receptors was negative, and he was started on palliative radiotherapy to the right groin, which included the primary disease, as well as the regional bony metastases. Unfortunately, the porocarcinoma did not respond to the palliative treatment, and he succumbed to the disease 2 years later.

The second case is a 56-year-old Malay gentleman, who was referred for a growth over the anterior chest. The lump was there since a young age, but the patient noticed that it was growing in size over the past few years and had occasional contact bleeding. Examination revealed a firm, solitary $6-\mathrm{mm}$ pigmented papule with central crusting over the suprasternal notch (Fig. 2a). There was no palpable lymphadenopathy or organomegaly.

Punch biopsy revealed proliferation of bland epithelial cells growing downwards into the underlying dermis, blending with sheets of epithelial cells, showing more atypical and pleomorphic nuclei with scattered mitotic activity. Immunohistochemical staining was positive for Ki-67. These features were consistent with porocarcinoma, supervening on background eccrine poroma.

A wide local excision with 1-cm margin was done. Histology revealed nests of cells with ovoid nuclei showing nuclear crowding and moderate nuclear pleomorphism (Fig. 2b). Duct formation and focal squamous differentiation was noted, with occasional mitoses. The excision margins were clear. As of last follow-up, a year after excision, there was no clinical evidence of recurrence.

Unusual Eccrine Porocarcinomas

\section{Discussion}

There is new evidence to suggest a 34 -fold increased risk of cutaneous SCCs for those diagnosed with cutaneous adnexal carcinomas [3]. Our first case interestingly demonstrates an occurrence of porocarcinoma after the diagnosis of SCC, suggesting the possibility of unknown risk factors for both. EPCs are not classically known to be related to sun exposure, and the occurrence of the tumour at the groin supports this finding. Positivity to androgen, oestrogen, and epidermal growth factor receptors was seen in a proportion of porocarcinomas [4], which, if positive, may allow for hormonal or targeted therapy. For now, there is paucity of data regarding treatment of metastatic porocarcinoma due to low case incidence. While our first case demonstrated good response to radiotherapy alone, further research can be done on combination therapy with hormone receptor antagonists.

Our second case demonstrates malignant change of a poroma. On histology, porocarcinoma shows irregular nests, and the cells may retain some features of a poroma (with ovoid nuclei and moderately abundant eosinophilic cytoplasm) and show ductal differentiation, reflecting its derivation from the intraepithelial ductal portion of eccrine sweat glands. However, there will be increased nuclear crowding, hyperchromasia, and slight nuclear pleomorphism, with increased mitotic activity in the case of porocarcinomas. Tumour cells are positive for epithelial membrane antigen, pan-cytokeratins, and p63 im- 
munostains. There is still little information on the incidence rate of malignant transformation of poromas [5] at present. Longstanding lumps on the body which experience any changes (such as rapid increase in size, ulceration, bleeding, or colour alteration) much later in life should prompt the dermatologist to investigate further, as there is a low threshold to rule out malignant transformation.

EPCs, although rare, are the most frequently encountered malignant eccrine gland tumour [2]. As both our cases demonstrate, EPCs can present in a myriad of ways. Specifically, EPCs can arise de novo or from malignant transformation of an existing poroma. Histologically, malignant porocarcinomas may express a greater variety of cellular markers than benign poromas, such as with CEA-positive single vacuolated cells observed in porocarcinomas, but not poromas [6]. Clinically, EPCs can present as nodules, infiltrative plaques, or ulcerated lesions. Differentials include squamous and basal cell carcinoma, amelanotic melanoma, and cutaneous metastasis of a visceral carcinoma [3]. Further research is needed particularly in the assessment of treatment response to guide the development of treatment recommendations.

\section{Acknowledgments}

The authors are grateful for the patients, who have so graciously offered their photos and their stories for the betterment of medical science.

\section{Statement of Ethics}

Ethical approval was not required for publishing of case reports fewer than 3 cases, as stated by the Singapore Institutional Review Boards. Written informed consent was obtained from both subjects for publication of the details of their medical case and any accompanying images.

\section{Conflict of Interest Statement}

The authors have no conflicts of interest to declare.

\section{Funding Sources}

No funding was needed for preparation of this manuscript.

\section{Author Contributions}

Lester Juay performed the literature review and wrote the manuscript. Ellie Choi performed the literature review and wrote parts of the manuscript. Jingxiang Huang provided the analysis and histological slides for both cases. Huma Jaffar provided clinical information and photos for the first case, while Sue-Ann Ju Ee Ho provided clinical information and photos for the second case and oversaw multiple revisions of the final manuscript.

\section{Data Availability Statement}

All data generated or analysed during this study are included in this article. Further enquiries can be directed to the corresponding author.

\section{References}

1 Riera-Leal L, Guevara-Gutiérrez E, Barrientos-García JG, Madrigal-Kasem R, BriseñoRodríguez G, Tlacuilo-Parra A. Eccrine porocarcinoma: epidemiologic and histopathologic characteristics. Int J Dermatol. 2015;54(5): 580-6.

2 De Giorgi V, Salvati L, Barchielli A, Caldarella A, Gori A, Scarfi F, et al. The burden of cutaneous adnexal carcinomas and the risk of associated squamous cell carcinoma: a population-based study. Br J Dermatol. 2019; 180(3):565-73.

3 Nazemi A, Higgins S, Swift R, In G, Miller K, Wysong A. Eccrine porocarcinoma: new in- sights and a systematic review of the literature. Dermatol Surg. 2018;44(10):1247-61.

4 Le LP, Dias-Santagata D, Pawlak AC, Cosper AK, Nguyen AT, Selim MA, et al. Apocrineeccrine carcinomas: molecular and immunohistochemical analyses. PLoS One. 2012; 7(10):e47290

5 Sawaya JL, Khachemoune A. Poroma: a review of eccrine, apocrine, and malignant forms. Int J Dermatol. 2014;53(9):1053-61.

6 Wollina U, Castelli E, Rülke D. Immunohistochemistry of eccrine poroma and porocarcinoma - more than acrosyringeal tumors? Recent Results Cancer Res. 1995;139:303-16. 